\title{
Sensitive and fast response graphite pressure sensor fabricated by a solvent-free approach
}

\author{
Toan Dinh ${ }^{1, *}$ Hoang-Phuong Phan ${ }^{1}$, Tuan-Khoa Nguyen ${ }^{1}$, Jarred Fastier-Wooller ${ }^{2}$, Abu Riduan Md Foisal, ${ }^{1}$ \\ Waail Mahmod Lafta ${ }^{2}$, Nam-Trung Nguyen ${ }^{1}$, Dzung Viet Dao ${ }^{1,2}$ \\ ${ }^{1}$ Queensland Micro- and Nanotechnology Centre, Griffith University, Queensland, Australia \\ ${ }^{2}$ Griffith School of Engineering, Griffith University, Queensland, Australia \\ *Email: toan.dinh@griffithuni.edu.au
}

\begin{abstract}
This work demonstrates a biodegradable resistive pressure sensor fabricated by a solvent-free, low cost but highly efficient method. The sensor shows a high sensitivity of $4.77 \% / \mathrm{kPa}$, a fast response of $0.3 \mathrm{~ms}$ and a relaxation time of 8 ms. The capability of the sensor attached to the human body and measuring human motion in real-time indicates its potential for human motion detection, touch displays and tactile sensing applications.
\end{abstract}

Keywords-Graphite on paper, resistive pressure sensor, wearable sensor, human motion detection.

\section{INTRODUCTION}

Resistive pressure sensors are of high interest in terms of design, implementation and low power consumption [1]. Up to date, these sensors have been successfully demonstrated with high sensitivity and fast response $[1,2]$. Nanomaterials such as graphene, silver nanowires and conductive foams have been used to construct flexible/stretchable and wearable pressure sensors for emerging applications, including pulse detection and touch displays [3].

However, the fabrication of resistive pressure sensors has typically involved complex processes such as lithography and chemical vapour deposition which require advanced processing facilities [1-3]; hence it raises the cost of such devices, especially at low scale production. Moreover, most fabrication processes have employed toxic solvent or chemicals which could lead to environmental issues [4]. In addition, conventional pressure sensors have typically achieved high sensitivity at low pressure ranges (e.g. $<5 \mathrm{kPa}$ ), while their sensitivity decreases significantly at higher pressure ranges [5]. Most of these sensors have also exhibited a relatively low response/relaxation time (e.g. $>100 \mathrm{~ms}$ ) [6].

Here we demonstrate a simple but effective approach to fabricating resistive pressure sensors with high sensitivity and fast response time. The graphite sensing layers were deposited on paper substrates by conventional drawing methods, which include two interdigitated electrodes and a conducting pad. The sensor shows excellent performance such as a high sensitivity of $4.77 \% / \mathrm{kPa}$, a fast response time of $0.3 \mathrm{~ms}$ and a relaxation time of $8 \mathrm{~ms}$. The sensor was able to detect human touching and the motion of a human finger, indicating the strong feasibility of using the sensor for human motion detection and other touch display applications.

\section{FABRICATION OF THE SENSOR}

\section{A. Fabrication process}

Figure 1a shows the fabrication process of the resistive pressure sensor. The first layer of the sensor was created by forming two interdigitated electrodes on a sheet of printing paper (A4, Staples) using a pencil (5B, Faber-Castell). A square shaded pencil graphite on paper (GOP) was also made as a second conductive layer. A $500 \mu \mathrm{m}$ thick acrylic elastomer layer (3M VHB Acrylic Foam Tape) was employed to assembly the sensor. The inset in Figure 1a shows the asfabricate pressure sensor, which indicates its flexibility and bendability; hence it is wearable.

\section{B. Material properties}

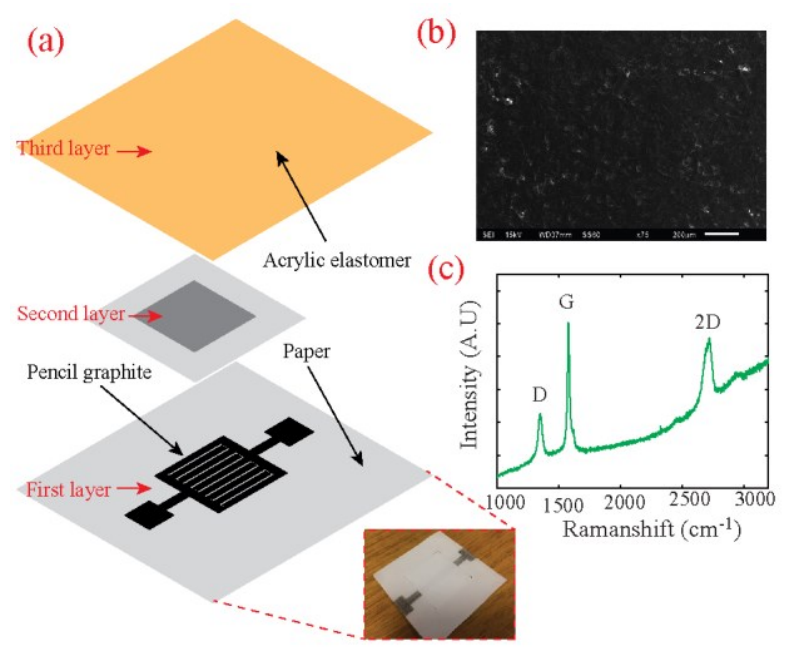

Figure 1: Fabrication of the sensor. (a) Fabrication process. The inset in Figure 1 shows the as-fabricated pressure sensor. (b) Scanning Electron Microscopy (SEM) image of the pencil graphite drawn on paper. (c) Raman spectrum of pencil graphite

Figure 1b shows a Scanning Electron Microscopy (SEM) image of the pencil graphite drawn on paper (GOP), which indicates a deposition of graphite on the porous paper substrate. To qualify the properties of the GOP material, we performed Raman measurement and the result is presented in Figure 1c. Three main peaks were observed at the wavenumbers of 1350,1580 and $2725 \mathrm{~cm}^{-1}$, corresponding to the $\mathrm{D}, \mathrm{G}$ and 2D bands of graphite materials, respectively. The intensity of G-band is much large than that of D-band, indicating the high quality of the graphite material [7]. 


\section{Sensing mechanism}

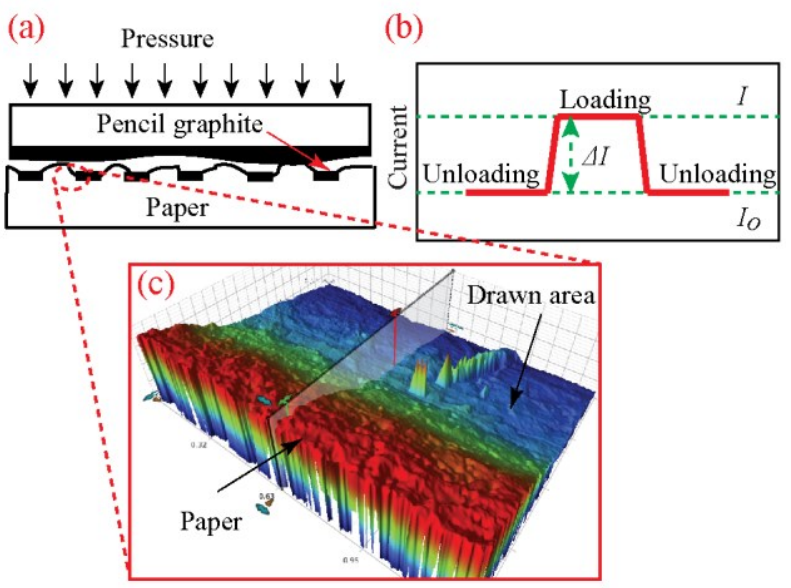

Figure 2: Sensing mechanism of the pressure sensor. (a) Schematic sketch of the sensing mechanism. (b) Concept of current variation under loading and unloading conditions. (c) A 3D surface profiling image of the GOP.

Figure 2a shows the schematic sketch of the sensor mechanism. The shaded area was deformed after the drawing process and the roughness of the surface led to a pressuredependent contact resistance between the graphite interdigitated electrodes and the conductive pad of the second GOP layer. Under a constant voltage mode, when a pressure is applied, the contact area increases, leading to an increase in the measured electrical current (Figure 2b). Figure 2c shows a 3D surface profiling image image of the GOP indicating a deformation of the shaded area.

\section{RESULTS AND DISCUSSION}

\section{A. Sensitivity}

The sensor was calibrated using a force and displacement control system and the current variation was simultaneously monitored using an USB measurement unit (Agilent Technologies) (see the inset in Figure 3).

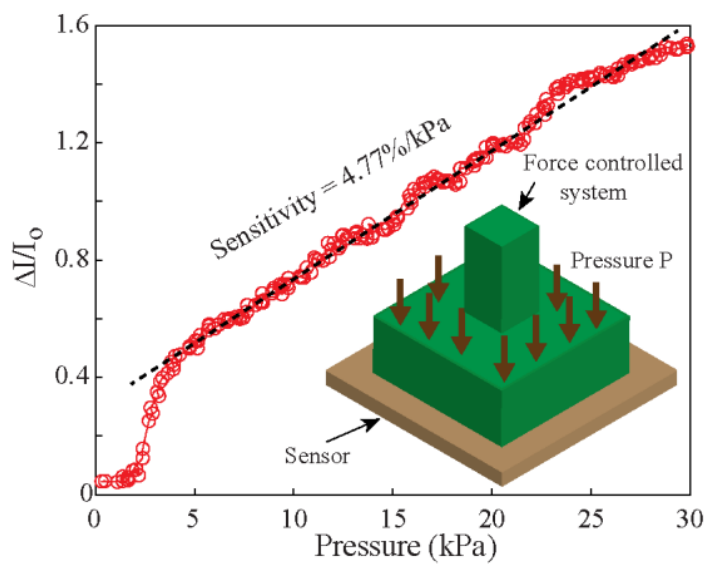

Figure 3: Sensor calibration

Figure 3 shows the change of the electrical current under various applied pressures. The linear characteristics of the sensor was observed at a pressure range of 5 to $30 \mathrm{kPa}$, which indicates a high sensitivity of $4.77 \% / \mathrm{kPa}$. The high sensitivity within the pressure range of 5 to $30 \mathrm{kPa}$ is of high interest for developing touch displays, flexible robotics and tactile sensing applications.

\section{B. Response and relaxation time}
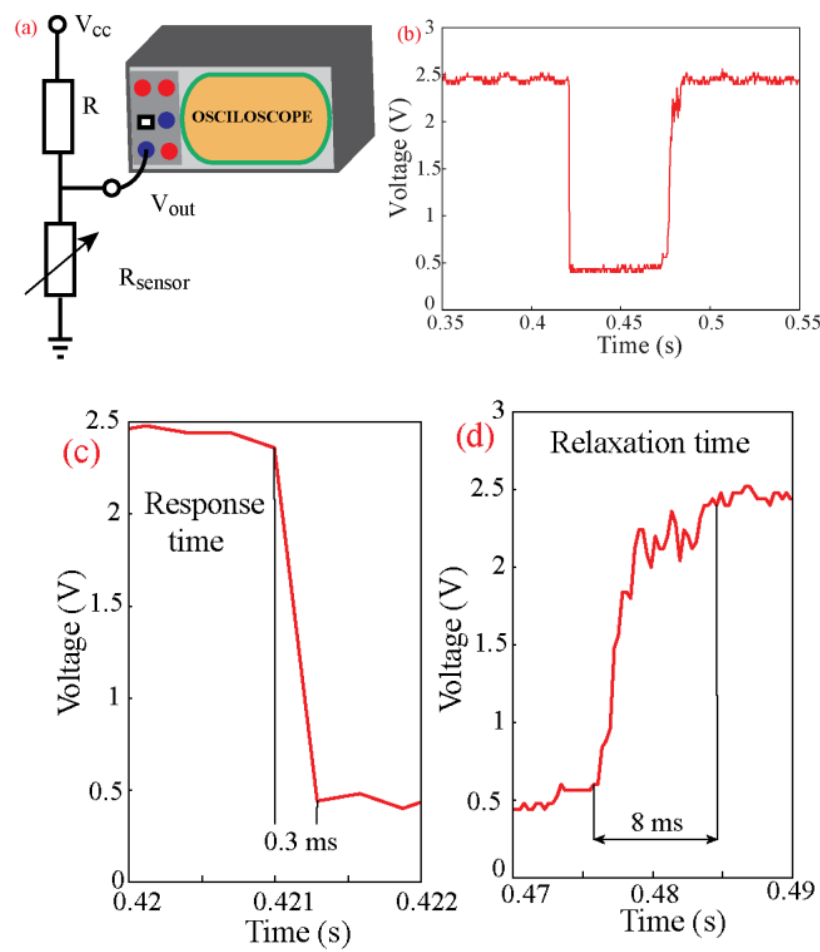

Figure 4: Response of the sensor. (a) Experimental setup to measure the response of the sensor. (b) The response of the sensor with loading and unloading conditions. (c) Response time of the sensor. (d) Relaxation time of the sensor.

Figure 4a shows the schematic sketch of the experimental setup for monitoring the response of the sensor. A constant voltage was applied on a series of an external resistor and the sensor, while the output voltage of the sensor was measured using an oscilloscope. Figure $4 \mathrm{~b}$ shows the response of the sensor under loading and unloading conditions. The close-up image for the response time is presented in Figure $4 \mathrm{c}$ which indicates a fast response time of $0.3 \mathrm{~ms}$. In addition, Figure $4 \mathrm{~d}$ shows the relaxation time of $8 \mathrm{~ms}$ for the sensor.

\section{Demonstrations for tactile and wearable sensing applications}

We demonstrate the application of the sensor for tactile sensing, touching displays and soft skins by applying finger touch on the sensor (e.g. gentle and hard touch). Figure 5 shows the response of the sensor to the finger touch, which indicates a good repeatability and high sensitivity of the sensor signal.

For wearable applications, we fabricated another pressure sensor with a dimension of $12 \mathrm{~mm} \times 80 \mathrm{~mm}$ for attaching on a finger. As the sensor was constructed on flexible paper substrate and acrylic elastomer, it offers wearable functionality as shown in the inset in Figure 6. Figure 6 shows the response 
of the sensor to the motion of human finger in real-time with high sensitivity and high signal-to-noise ratio. This indicates the possibility of using the sensor for human motion detection and other wearable applications.

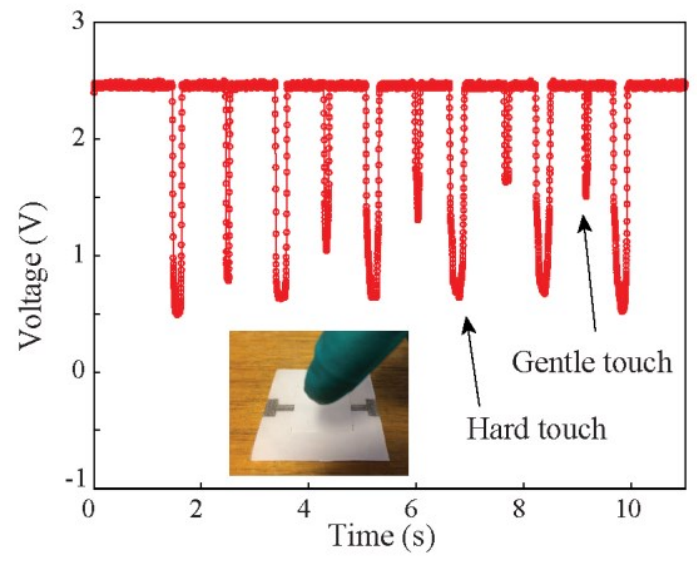

Figure 5: Demonstration of tactile sensing

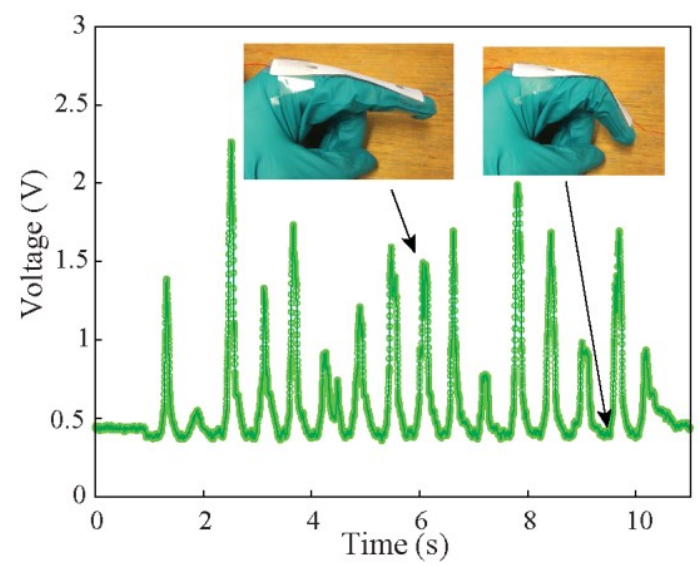

Figure 6: Demonstration for human motion detection

\section{CONCLUSION}

We successfully fabricated an ultra-low cost, highly sensitive and fast response resistive pressure sensor using a solvent-free fabrication process. The sensor showed a high sensitivity of $4.77 \% / \mathrm{kPa}$ for a pressure range of 5 to $30 \mathrm{kPa}$, and exhibited a fast response time of $0.3 \mathrm{~ms}$ and a relaxation time of $8 \mathrm{~ms}$. The sensor was able to detect finger touch and motion in real-time, demonstrating a strong feasibility of using this sensor for tactile sensing, touch displays and wearable applications.

\section{ACKNOWLEDGMENT}

This work was performed in part at the Queensland node of the Australian National Fabrication Facility, a company established under the National Collaborative Research Infrastructure Strategy to provide nano and micro-fabrication facilities for Australia's researchers. This work has been partly supported by the Australian Research Council grant LP160101553.

\section{REFERENCES}

[1] S. Gong, W. Schwalb, Y. Wang, Y. Chen, Y. Tang, J. Si, et al., "A wearable and highly sensitive pressure sensor with ultrathin gold nanowires," Nature communications, vol. 5, 2014.

[2] L. Pan, A. Chortos, G. Yu, Y. Wang, S. Isaacson, R. Allen, et al., "An ultra-sensitive resistive pressure sensor based on hollow-sphere microstructure induced elasticity in conducting polymer film," Nature communications, vol. 5, 2014.

[3] C. L. Choong, M. B. Shim, B. S. Lee, S. Jeon, D. S. Ko, T. H. Kang, et al., "Highly stretchable resistive pressure sensors using a conductive elastomeric composite on a micropyramid array," Advanced Materials, vol. 26, pp. 3451-3458, 2014.

[4] C. Pang, J. H. Koo, A. Nguyen, J. M. Caves, M. G. Kim, A. Chortos, et al., "Highly Skin Conformal Microhairy Sensor for Pulse Signal Amplification," Advanced materials, vol. 27, pp. 634-640, 2015.

[5] H. Tian, Y. Shu, X.-F. Wang, M. A. Mohammad, Z. Bie, Q.-Y. Xie, et al., "A graphene-based resistive pressure sensor with record-high sensitivity in a wide pressure range," Scientific reports, vol. 5, 2015.

[6] S. Brady, D. Diamond, and K.-T. Lau, "Inherently conducting polymer modified polyurethane smart foam for pressure sensing," Sensors and Actuators A: Physical, vol. 119, pp. 398-404, 2005.

[7] T. Dinh, H.-P. Phan, D. V. Dao, P. Woodfield, A. Qamar, and N.-T. Nguyen, "Graphite on paper as material for sensitive thermoresistive sensors," Journal of Materials Chemistry C, vol. 3, pp. 8776-8779, 2015. 